\title{
The availability of Interventional Radiology for the insertion of central venous access devices in NHS Scotland
}

\author{
J. P. Baxter ${ }^{1}$, R. F. McKee ${ }^{2}$, I. Zealley ${ }^{3}$ and A. W. McKinlay ${ }^{4}$ \\ ${ }^{1}$ Scottish Home Parenteral Nutrition Managed Clinical network, ${ }^{2}$ Department of Surgery, Glasgow Royal Infirmary, \\ ${ }^{3}$ Department of Radiology Ninewells Hospital and Medical School, Dundee and ${ }^{4}$ Departmant of Gastroenterology, \\ Aberdeen Royal Infirmary, UK
}

There is evidence that using ultrasound guidance is a more effective method of insertion in adults (Bishop et al. 2007, SHPNMCN 2007). The purpose of this audit was to identify the levels of access that nutrition support teams in Scotland have to radiological services and if the service was routinely used.

Each of the centres managing HPN patients was e-mailed to ask if they had access to, and used, interventional radiology (IR) for insertion of central venous access devices (CVAD). Responses were cross checked with data from a previous IR audit.

All centres managing HPN patients responded to the request for information. All of the paediatric centres reported that a paediatric surgeon carries out CVAD insertion. Surgeons place the catheters in Edinburgh using ultrasound guidance and percutaneous placement in all but the very smallest babies. This is essentially the same techniques as the interventional radiologists. The table below shows the HPN centres in NHS Scotland Board, and if radiology is used for CVAD insertion.

\begin{tabular}{lll}
\hline HPN Centre (Adult care) & \multicolumn{1}{c}{ NHS Board } & Access to IR \\
\hline Ninewells Hospital & Tayside & Yes \\
Glasgow Royal Infirmary, Western Infirmary, & Greater Glasgow and Clyde & Yes \\
Gartnavel, Southern General & Grampian & Yes (with waiting list) \\
Aberdeen Royal Infirmary & Ayrshire and Arran & Yes \\
Crosshouse Hospital, Kilmarnock & Fife & Yes \\
Queen Margaret Hospital, Dunfermline & Highland & Yes \\
Raigmore Hospital, Inverness, & Lothian & Yes \\
Western General Hospital, Edinburgh, & & \\
\hline
\end{tabular}

Information from radiology reported IR being available in NHS Forth Valley and Lanarkshire, and could theoretically insert CVADs, but not available in Borders, Dumfries and Galloway, Orkney, Shetland or Western Isles.

All centres managing HPN patients at the time of the audit responded. Several NHS Boards do not have IR, but we know that these boards rarely have HPN patients, and if they do, patients are referred an HPN centres, via service agreement or inter departmental referral. The response that IR is not used in paediatric HPN centres, reflects paediatric practice. This is important data to confirm availability of the optimal method of CVAD insertion. The issue of waiting list was escalated within NHS Grampian.

1. Bishop L, Dougherty L, Bodenham A, Mansi J, Crowe P, Kibbler C, Shannon M \& Treleaven J (2007) Guidelines on the insertion and management of central venous access devices in adults. International Journal of Laboratory Hematology 29(4):261-278.

2. Clinical Standards for Home Parenteral Nutrition. (2007) SHPNMCN. 